\title{
Clinical presentation does not affect acute mechanical performance of the Novolimus-eluting bioresorbable vascular scaffold as assessed by optical coherence tomography
}

\author{
Niklas F. Boeder ${ }^{1}$, Oliver Dörr ${ }^{1}$, Rosalina Gaderer ${ }^{1}$, Florian Blachutzik ${ }^{1}$, Stephan Achenbach ${ }^{2}$, \\ Albrecht Elsässer ${ }^{3}$, Christian Hamm ${ }^{1}$, Holger M. Nef ${ }^{1}$ \\ ${ }^{1}$ Department of Cardiology, University of Gießen, Gießen, Germany \\ ${ }^{2}$ Department of Cardiology, University of Erlangen, Erlangen, Germany \\ ${ }^{3}$ Department of Cardiology, Oldenburg, Germany
}

Adv Interv Cardiol 2021; 17, 3 (65): 272-280

DOI: https://doi.org/10.5114/aic.2021.109239

\begin{abstract}
Introduction: Initial trials of bioresorbable vascular scaffolds (BVS) have mostly excluded patients presenting with acute coronary syndrome (ACS). However, these patients might benefit from a BVS platform, in particular as they are often younger and have been less frequently treated than patients with chronic disease.

Aim: To compare the acute performance of a Novolimus eluting BVS in ACS and non-ACS patients using optical coherence tomography $(\mathrm{OCT})$ in patients presenting with acute or chronic coronary syndrome.

Material and methods: The final OCT pullback of 79 patients (34 with ACS, 45 non-ACS) was analysed at 1-mm intervals. The following indices were calculated: mean and minimal area, residual area stenosis, incomplete strut apposition, tissue prolapse, eccentricity index, symmetry index, strut fracture, and edge dissection.

Results: OCT showed a minimum lumen area (non-ACS vs. ACS) of $6.2 \pm 2.1$ vs. $5.6 \pm 1.5 \mathrm{~mm}^{2}(p=0.21)$. Mean residual area stenosis was $14.5 \%$ vs. $19.5 \%(p=0.39)$. The mean eccentricity index did not differ significantly $(0.78 \pm 0.13$ vs. $0.78 \pm 0.06 ; p=$ $0.42)$. There was a non-significant tendency for more fractures in the non-ACS group ( $22.2 \%$ vs. $5.9 \% ; p=0.07)$. Prolapse area was comparable $\left(4.4 \pm 7.4 \mathrm{~mm}^{2}\right.$ vs. $\left.5.2 \pm 10.9 \mathrm{~mm}^{2} ; p=0.62\right)$.

Conclusions: This is the first study to investigate the acute mechanical performance of a Novolimus-eluting BVS in patients with different clinical presentations using OCT. We found that clinical presentation did not determine acute mechanical performance as assessed by the final OCT pullback. There was evidence of more mechanical complications in terms of fractures and a higher percentage of incomplete strut apposition in the group of patients with chronic coronary syndrome.
\end{abstract}

Key words: bioresorbable vascular scaffold, optical coherence tomography, acute coronary syndrome.

Su m m a ry

The most widely investigated poly-L-lactic acid BRS, the Absorb BVS, was withdrawn from the market due to concerns that were mainly driven by an unexpected high thrombogenicity of bioresorbable vascular scaffolds (BVS) compared with drug-eluting stents. In addition, the Novolimus-eluting DESolve (Elixir Medical Corporation, Sunnyvale, CA, USA) was introduced. This is the first study to investigate the acute mechanical performance of a Novolimus-eluting BVS in patients with different clinical presentations using optical coherence tomography (OCT). We found that clinical presentation did not determine acute mechanical performance as assessed by the final OCT pullback. There was evidence of more mechanical complications in terms of fractures and a higher percentage of incomplete strut apposition in the group of patients with chronic coronary syndrome.

Corresponding author:

Dr. Niklas Frederik Boeder, Department of Cardiology, University of Gießen, Gießen, Germany, e-mail: niklas.boeder@innere.med.uni-giessen.de Received: 16.04.2021, accepted: 29.06.2021. 


\section{Introduction}

Bioresorbable vascular scaffolds (BVS) are the latest innovation in the field of interventional cardiology for the treatment of patients with coronary syndrome. They were introduced into routine clinical practice to overcome limitations such as permanent caging by the device and chronic inflammation. Because they dissolve after several years, BVS offer transient vessel support. Thus, they allow and support the restoration of vasomotor function and also facilitate future surgical revascularization [1]. Whereas initial studies of outcome comparing BVS with drug-eluting stents (DES) showed promising results, randomized clinical trials and meta-analyses raised concerns regarding adverse events due to higher early and late thrombogenicity of BVS compared with DES for the treatment of de novo lesions [2, 3]. These findings were also consistently observed in so-called "real-world" registries [4]. Ultimately, the most widely investigated poly-L-lactic acid (PLLA) BRS, the Absorb BVS (Abbott Vascular, Santa Clara, CA, USA), was withdrawn from the market. The causes of scaffold thrombosis (ScT) are multifactorial. However, the notably high rates of early ScT soon pointed towards periprocedural aspects that contribute to its occurrence $[4,5]$. Hence, adaptation of the implantation technique and increased experience of the operator led to lower thrombosis rates. Furthermore, the lesion type and the clinical presentation of the patient appear to play a role in BVS performance [6]. It has been shown that the risk of device thrombosis after percutaneous coronary intervention $(\mathrm{PCl})$ is increased in patients with acute coronary syndrome (ACS). Given the greater strut thickness of BVS to compensate for a decreased radial strength, concerns have been raised with regard to ScT in BVS as this might increase shear stress and flow disturbance, especially in the hypercoagulable setting of ACS. Therefore, ACS has been an exclusion criterion for most of the randomized trials, although it is an appealing scenario for BVS: younger patients, fewer prior events, and softer plaque. In addition, the Novolimus-eluting DESolve (Elixir Medical Corporation, Sunnyvale, CA, USA) was introduced.

\section{Aim}

In the present study we investigated the acute mechanical device properties of the Novolimus-eluting DESolve BVS in patients who underwent implantation and compared these parameters with the patients' clinical presentation.

\section{Material and methods}

Consecutive patients who underwent $\mathrm{PCl}$ with Novolimus-eluting BVS were enrolled in this retrospective study irrespective of their clinical presentation. The index procedure was carried out between January 2014 and Au- gust 2015. Patients were classified into ACS and non-ACS groups. Here ACS was defined as ST-elevation myocardial infarction (STEMI), non-ST-elevation myocardial infarction (NSTEMI), or unstable angina.

The DESolve BVS is a polymeric device with a strut thickness of $150 \mu \mathrm{m}$ that elutes Novolimus. It has greater radial strength and a faster degradation process than the Absorb BVS, and it can be overexpanded up to $0.5 \mathrm{~mm}$ above the nominal diameter. Bench tests even showed a higher overexpansion capacity during post-dilatation without increasing fractures [7]. Furthermore, the DESolve BVS is able to self-correct for minor malapposition [8].

$\mathrm{PCl}$ was performed in a single, high-volume centre in accordance with standard clinical practice. The radial approach using a 6 French guiding catheter was favoured and used whenever feasible. At the beginning of the procedure all patients were administered $70 \mathrm{U} / \mathrm{kg}$ body weight unfractionated heparin. Prior to lesion preparation patients were administered intracoronary nitroglycerine. Lesion preparation was initiated with pre-dilatation utilizing a non-compliant balloon that corresponded $1: 1$ to vessel size. The use of a debulking device was left to the operator's discretion. Similar to balloon sizing, BVS sizing was approximately $1: 1$ with respect to the vessel diameter. Its deployment was accomplished by slowly inflating the stent balloon ( $1 \mathrm{~atm}$ over $10 \mathrm{~s}, 2 \mathrm{~atm}$ over $10 \mathrm{~s}$, then $2 \mathrm{~s}$ per atm). The final pressure was maintained for 20 s. Post-dilatation was performed with a non-compliant balloon in accordance with the maximum expansion limits of the BVS.

Frequency domain optical coherence tomography (OCT) was performed using the llumien Optis system (St. Jude Medical, Inc., Minneapolis, MN, USA). Pullbacks were performed in an automatic manner or manually, if necessary, at $36 \mathrm{~mm} / \mathrm{s}$ during contrast injection at a rate of 3 to $5 \mathrm{ml} / \mathrm{s}$. After having placed the imaging catheter distally to the treated lesion, the pullback was recorded until either the guiding catheter was reached or the maximum pullback length was completed. In the latter case, or if the treated lesion was incompletely recorded, a second sequential pullback was combined to image the whole lesion. Data from the final pullback just before the end of the procedure were used for the analysis in this study.

OCT measurements were performed offline using the LightLab Imaging workstation (St. Jude Medical, Inc.). Longitudinal cross-sections of the final pullback were analysed at 1-mm intervals within the stented lesion and $5 \mathrm{~mm}$ proximally and distally to the scaffold (Figure 1). The following quantitative parameters were determined manually: the percentage of incomplete strut apposition (ISA) at 1-mm intervals, calculated as a percentage of the total number of malapposed struts divided by the total number of struts (Figure 2); the ISA area; the tissue prolapse area (Figure 3), defined as the projection of tissue 


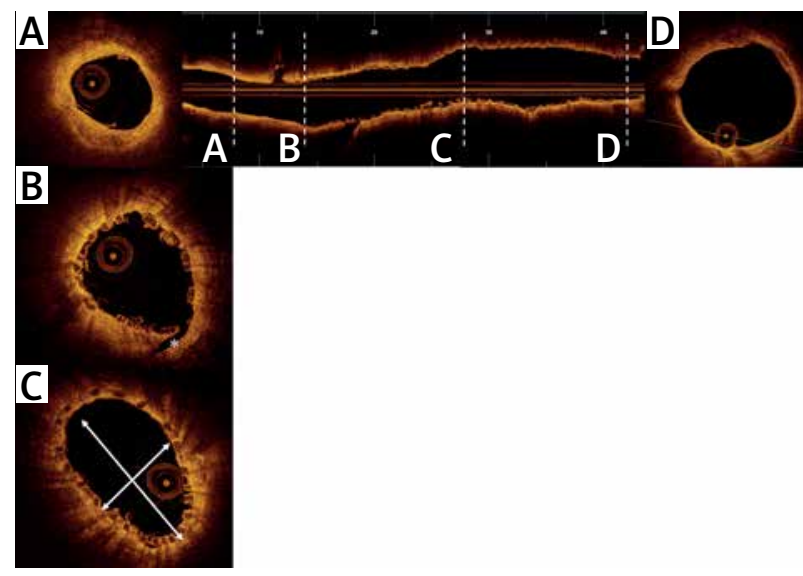

Figure 1. A - Distal reference vessel area (DRVA) $=3.77 \mathrm{~mm}^{2}, \mathbf{B}-$ asterisk indicating a distal edge dissection, $\mathbf{C}$ - cross section with minimum eccentricity index (minimum/maximum diameter) $=$ $(2.29 \mathrm{~mm} / 3.96 \mathrm{~mm})=0.57, \mathrm{D}$ - proximal reference vessel area $(P R V A)=11.59 \mathrm{~mm}^{2}$. Reference vessel area $(R V A)=(P R V A+D R V A) / 2=\left(11.59 \mathrm{~mm}^{2}\right.$ $\left.+3.37 \mathrm{~mm}^{2}\right) / 2=7.48 \mathrm{~mm}^{2}$

into the lumen between struts; residual area stenosis (RAS), calculated as (1-minimum lumen area (MLA)/reference vessel area (RVA)); the eccentricity index, computed as the ratio between the minimum and maximum diameters; the symmetry index, defined as the difference between maximum scaffold diameter and minimum scaffold diameter divided by the maximum scaffold diameter. If no meaningful value for proximal or distal RVA was obtained, the largest luminal cross-sectional area at the distal or proximal end of the scaffold was used. An edge dissection was defined as any disruption of the vessel luminal surface at the edges of the scaffold with a visible flap $(>300 \mu \mathrm{m})$. A scaffold fracture was assumed if isolated struts were observed to be unopposed within the scaffold lumen or if struts were stacked. The analysis of the OCT pullbacks was conducted by the local core lab for intravascular imaging.

Quantitative coronary angiography (QCA) was carried out with the help of offline QCA software (CAAS QCA, Pie Medical Imaging BV, The Netherlands). The following parameters were assessed during post-hoc analysis: reference vessel diameter (RVD) through automatic interpolation, minimum lumen diameter (MLD), percentage area stenosis (AS), and lesion length. Analysis was independently performed by two experienced interventional cardiologists.

\section{Statistical analysis}

Statistical analysis was performed using IBM SPSS Statistics (SPSS Statistics 23, IBM Deutschland GmbH, Ehningen, Germany). Continuous variables with normal distribution are expressed as means and standard de-

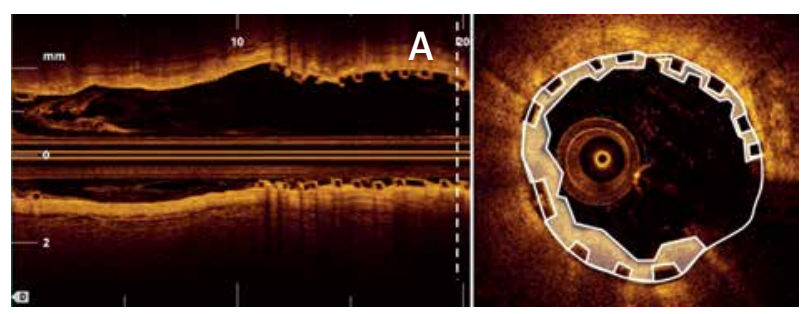

Figure 2. Prolapse area $=$ scaffold area - lumen area : $4.95 \mathrm{~cm}^{2}-3.38 \mathrm{~cm}^{2}=1.57 \mathrm{~cm}^{2}$

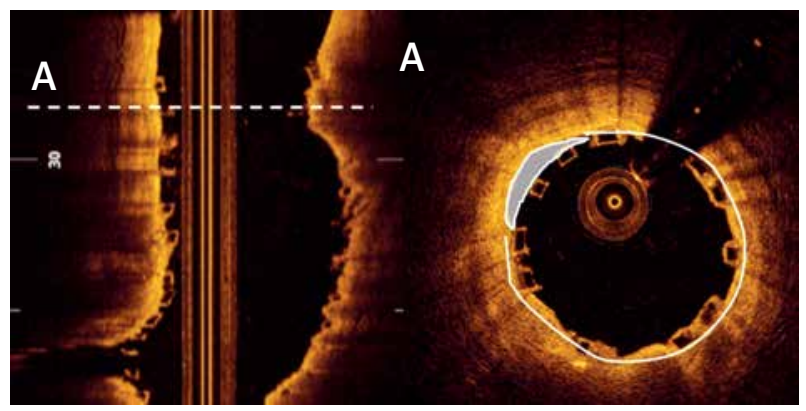

Figure 3. Malapposition = lumen area - scaffold area: $6.73 \mathrm{~cm}^{2}-6.45 \mathrm{~cm}^{2}=0.28 \mathrm{~cm}^{2}$

viations; categorical variables are given as number and percent. The $\chi^{2}$ and Fisher's exact tests were used for comparison of categorical variables, and Student's $t$-test or the Wilcoxon rank-sum test was applied for continuous variables. $P$-values $<0.05$ were considered statistically significant.

\section{Results}

A total of 79 patients were enrolled in this study. All patients were implanted with a Novolimus-eluting BVS. Thirty-four patients presented with ACS, and 45 patients were diagnosed with chronic coronary syndrome (non-ACS group). Patients in the ACS group were significantly younger (non-ACS $64.9 \pm 8.5$ years vs. ACS 57.4 \pm 7.5 years, $p<0.001$ ) and less frequently treated for arterial hypertension ( $100 \%$ vs. $79.4 \%, p<0.001)$. Patients in the two groups had a similar prevalence of diabetes mellitus $(13.3 \%$ vs. $25.5 \%, p=0.15)$ and smoking $(58 \%$ vs. $70 \%, p=0.24)$ as well as a similar left ventricular ejection fraction ( $56.1 \pm 95$ vs. $53.8 \pm 10.5 \%, p=0.39)$ and number of diseased vessels (Table I).

Lesions were typically located in the RCA in the nonACS and the LAD in the ACS group $(p=0.01)$. They did not differ significantly with respect to AHA/ACC lesion classification (Table II). In most cases lesions were classified as being of type B1 (35.6 vs. $43.8 \%$ for non-ACS vs. ACS, respectively). QCA analysis showed no difference between the two groups with respect to reference vessel diameter ( $2.6 \pm 0.7 \mathrm{~mm}$ vs. $2.4 \pm 0.5 \mathrm{~mm} ; p=0.43)$, area stenosis (74.5\% vs. $74.4 \% ; p=0.89)$ and lesion length $(10.6 \pm 4.5 \mathrm{~mm}$ vs. $10.0 \pm 5.0 \mathrm{~mm} ; p=0.48)$. 
Table I. Baseline characteristics

\begin{tabular}{|c|c|c|c|}
\hline Parameter & Non-ACS $(n=45)$ & $\operatorname{ACS}(n=34)$ & $P$-value \\
\hline Age [years] & $64.9 \pm 8.5$ & $57.4 \pm 7.5$ & $<0.001^{\star}$ \\
\hline Female sex (\%) & 60.0 & 67.6 & 0.46 \\
\hline Hypertension (\%) & 100 & 79.4 & $<0.001^{*}$ \\
\hline Hyperlipoproteinemia (\%) & 75.6 & 52.9 & $0.04^{\star}$ \\
\hline Diabetes mellitus (\%) & 13.3 & 26.5 & 0.15 \\
\hline Current smoker (\%) & 57.8 & 70.6 & 0.24 \\
\hline Family history (\%) & 33.3 & 41.2 & 0.47 \\
\hline Prior PCI (\%) & 35.6 & 14.7 & $0.04^{*}$ \\
\hline Prior MI (\%) & 42.2 & 8.8 & $<0.001^{*}$ \\
\hline Left ventricular ejection fraction (\%) & $56.1 \pm 9.5$ & $53.8 \pm 10.5$ & 0.39 \\
\hline Clinical indication (\%): & & & $<0.001^{*}$ \\
\hline Stable angina & 100 & 0 & \\
\hline STEMI & 0 & 35.3 & \\
\hline NSTEMI & 0 & 17.1 & \\
\hline Unstable angina & 0 & 47.1 & \\
\hline Number of diseased vessels (\%): & & & 0.46 \\
\hline 1 & 20 & 23.5 & \\
\hline 2 & 22.2 & 32.4 & \\
\hline 3 & 57.8 & 44.1 & \\
\hline
\end{tabular}

*Significant difference $p<0.05 . P C l$ - percutaneous coronary intervention, MI - myocardial infarction, STEMI - ST-elevation myocardial infarction, NSTEMI non-ST-elevation myocardial infarction, ACS - acute coronary syndrome.

Table II. Angiographic and QCA lesions' characteristics

\begin{tabular}{|c|c|c|c|}
\hline Parameter & Non-ACS $(n=45)$ & $\operatorname{ACS}(n=34)$ & $P$-value \\
\hline Target vessel (\%): & & & $0.01^{*}$ \\
\hline LAD & 22.2 & 52.9 & \\
\hline$\overline{L C X}$ & 24.4 & 20.6 & \\
\hline RCA & 53.3 & 26.5 & \\
\hline AHA/ACC lesion classification (\%): & & & 0.70 \\
\hline Type A & 26.7 & 31.3 & \\
\hline Type B1 & 35.6 & 43.8 & \\
\hline Type B2 & 24.4 & 15.6 & \\
\hline Type C & 13.3 & 9.4 & \\
\hline \multicolumn{4}{|l|}{ QCA analysis: } \\
\hline $\mathrm{RVD}[\mathrm{mm}]$ & $2.6 \pm 0.7$ & $2.4 \pm 0.5$ & 0.43 \\
\hline $\mathrm{MLD}[\mathrm{mm}]$ & $1.2 \pm 0.5$ & $1.1 \pm 0.4$ & 0.15 \\
\hline AS (\%) & 74.5 & 74.7 & 0.89 \\
\hline Lesion length [mm] & $10.6 \pm 4.5$ & $10.0 \pm 5.0$ & 0.48 \\
\hline
\end{tabular}

*Significant difference $p<0.05$. LAD - left anterior descending artery, RCX - left circumflex artery, AHA - American Heart Association, ACC - American College of Cardiology, QCA - quantitative coronary angiography, RVD - reference vessel diameter, MLD - minimal lumen diameter, AS - area stenosis, ACS - acute coronary syndrome.

Lesion preparation was initiated with pre-dilatation in most cases in both groups (93.3\% vs. $97.1 \% ; p=0.46)$. Usage of non-compliant balloons was comparable in the two groups ( $81 \%$ vs. $75.8 \% ; p=0.58)$. There was no difference between the groups with respect to maximal pressure applied during pre-dilatation or the size of the balloon used (Table III). The implanted scaffold mean diameter was $3.1 \pm 0.4 \mathrm{~mm}$ vs. $3.1 \pm 0.4 \mathrm{~mm}(p=0.89)$ and the mean length was $19.7 \pm 5.9 \mathrm{~mm}$ vs. $19.8 \pm 5.6 \mathrm{~mm} \mathrm{(} p=$ $0.73)$. The deployment pressure applied did not differ be- tween the non-ACS and ACS groups. In accordance with the concept of routine pre-dilatation, final post-dilatation was performed in most cases ( $80 \%$ vs. $88.2 \%$; $p=0.33$ ). Diameter, length, and inflation pressure were similar in the two groups (Table III).

OCT findings are summarized in Table IV. A total of 20,008 struts and 1,593 cross-sections were analysed. Final mean and maximum scaffold diameters were similar in the non-ACS and ACS groups (mean: $3.1 \pm 0.4 \mathrm{~mm}$ vs. $3.0 \pm 0.4 \mathrm{~mm}$; $p=0.11$; maximum: $3.5 \pm 0.5 \mathrm{~mm}$ vs. 3.4 
Table III. Procedural characteristics

\begin{tabular}{lccc} 
Parameter & Non-ACS $(\boldsymbol{n}=\mathbf{4 5})$ & ACS $(\boldsymbol{n}=\mathbf{3 4 )}$ & $\boldsymbol{P}$-value \\
\hline Pre-dilatation (\%) & 93.3 & 97.1 & 0.46 \\
\hline Pre-dilatation with NC (\%) & 81.0 & 75.8 & 0.58 \\
\hline Max. diameter balloon pre-dilatation [mm] & $3.0 \pm 0.5$ & $2.9 \pm 0.4$ & 0.74 \\
\hline Max. pre-dilatation balloon length [mm] & $15.9 \pm 3.9$ & $15.4 \pm 3.3$ & 0.56 \\
\hline Max. pre-dilatation balloon inflation [atm] & $13.5 \pm 2.8$ & $13.9 \pm 3.5$ & 0.54 \\
\hline Scaffold diameter [mm] & $3.1 \pm 0.4$ & $3.1 \pm 0.4$ & 0.89 \\
\hline Scaffold length [mm] & $19.7 \pm 5.9$ & $19.8 \pm 5.6$ & 0.73 \\
\hline Scaffold deployment pressure [atm] & $13.9 \pm 2.4$ & $12.9 \pm 2.5$ & 0.09 \\
\hline Post-dilatation (\%) & 80 & 88.2 & 0.33 \\
\hline Max. post-dilatation balloon diameter [mm] & $3.7 \pm 0.7$ & $3.5 \pm 0.5$ & 0.18 \\
\hline Max. post-dilatation balloon length [mm] & $14.3 \pm 3.3$ & $16.5 \pm 4.1$ & 0.05 \\
\hline Max. post-dilatation balloon inflation [atm] & $16.3 \pm 3.5$ & $16.8 \pm 3.6$ & 0.39 \\
\hline Post-dilatation with NC (\%) & 75.6 & 88.2 & 0.19
\end{tabular}

NC-non-compliant, ACS - acute coronary syndrome.

Table IV. Optical coherence tomography findings

\begin{tabular}{|c|c|c|c|}
\hline Parameter & Non-ACS $(n=45)$ & $\operatorname{ACS}(n=34)$ & $P$-value \\
\hline Mean scaffold area $\left[\mathrm{mm}^{2}\right]$ & $8.1 \pm 2.3$ & $7.2 \pm 1.9$ & 0.06 \\
\hline Mean scaffold diameter [mm] & $3.1 \pm 0.4$ & $3.0 \pm 0.4$ & 0.11 \\
\hline Minimum scaffold diameter [mm] & $2.8 \pm 0.4$ & $2.6 \pm 0.4$ & 0.09 \\
\hline Maximum scaffold diameter [mm] & $3.5 \pm 0.5$ & $3.4 \pm 0.4$ & 0.34 \\
\hline Mean lumen area $\left[\mathrm{mm}^{2}\right]$ & $7.8 \pm 2.3$ & $7.1 \pm 1.8$ & 0.28 \\
\hline Minimum lumen area $\left[\mathrm{mm}^{2}\right]$ & $6.2 \pm 2.1$ & $5.6 \pm 1.5$ & 0.21 \\
\hline Percentage RAS (\%) & 14.5 & 19.5 & 0.39 \\
\hline Scaffold with RAS > 20\% (\%) & 42.2 & 52.9 & 0.34 \\
\hline Mean eccentricity index & $0.78 \pm 0.13$ & $0.78 \pm 0.06$ & 0.42 \\
\hline Minimum eccentricity index & $0.64 \pm 0.10$ & $0.63 \pm 0.09$ & 0.31 \\
\hline Symmetry index & $0.42 \pm 0.10$ & $0.42 \pm 0.09$ & 0.97 \\
\hline \multicolumn{4}{|l|}{ ISA: } \\
\hline ISA area $\left[\mathrm{mm}^{2}\right]$ & $1.3 \pm 2.7$ & $0.7 \pm 1.6$ & 0.17 \\
\hline Percentage of malapposed struts (\%) & 2.7 & 1.9 & 0.15 \\
\hline Prolapse area $\left[\mathrm{mm}^{2}\right]$ & $4.4 \pm 7.4$ & $5.2 \pm 10.9$ & 0.62 \\
\hline Strut fracture (\%) & 22.2 & 5.9 & 0.07 \\
\hline Edge dissection: & & & 0.50 \\
\hline Proximal edge (\%) & 2.2 & 5.9 & \\
\hline Distal edge (\%) & 4.4 & 0 & \\
\hline
\end{tabular}

RAS - residual area stenosis, ISA - incomplete strut apposition, ACS - acute coronary syndrome.

$\pm 0.4 \mathrm{~mm} ; p=0.34)$. Likewise, the mean scaffold area and mean lumen area did not differ between the two groups (Table IV). The mean residual area stenosis was $14.5 \%$ in patients presenting with a chronic coronary syndrome and $19.5 \%$ in patients presenting with ACS $(p=0.39)$. Assessment of geometric parameters revealed a mean eccentricity index of $0.78 \pm 0.13$ vs. $0.78 \pm 0.06(p=0.42)$ and a symmetry index of $0.42 \pm 0.10$ vs. $0.42 \pm 0.09$ ( $p=$ 0.97). OCT showed only a few dissections that occurred similarly in both groups ( $6.6 \%$ vs. $5.9 \% ; p=0.50)$. Fractures, however, tended to occur more frequently in the non-ACS group (22.2 vs. 5.9\%), although this difference was not significant $(p=0.07)$. The percentage of malap- posed struts was 2.7 vs. $1.9 \%(p=0.15)$. OCT analysis revealed a slightly, although not significantly, greater area in the ACS group (2.7\% vs. $1.9 \% ; p=0.15)$.

No adverse event occurred within the ensuing post-procedural period. In particular, no scaffold thrombosis was documented.

\section{Discussion}

This is the first study to compare the acute mechanical performance of a Novolimus-eluting BVS with clinical presentation by means of OCT. The patient collective comprised two groups: those presenting with ACS and those with chronic coronary syndrome. The principal 
findings are that: 1) good acute mechanical performance of the device was noted irrespective of the initial clinical presentation of the patient; and 2) patients with chronic coronary syndrome tended to have more mechanical complications, e.g. scaffold fractures and ISA.

The study population fulfilled the typical criteria for the implantation of BVS. This holds true especially for patients with ACS, who were relatively young and had a short history of coronary disease. Furthermore, the lesion complexity, as assessed using the criteria of the American College of Cardiology/American Heart Association [9], was predominantly simple and none of the cases involved a bifurcation.

The study shows that Novolimus-eluting BVS can be implanted with good acute mechanical performance in both groups. This finding is based on our measurement of parameters derived from previous studies that investigated the success of stent deployment $[10,11]$, which predominantly made use of intravascular ultrasound (IVUS) to evaluate acute and long-term clinical outcome in patients who were treated with either bare metal stents or first-generation DES $[12,13]$. The authors found that a minimum cross-sectional lumen area $<5.5 \mathrm{~mm}^{2}$ and an in-scaffold RAS > 20\% increases the risk of stent thrombosis. Patients treated in our cohort showed an average RAS of $14.5 \%$ in the non-ACS group and $19 \%$ in the ACS group, values that are not significantly different $(p=0.39)$. The number of scaffolds that showed an RAS $>20 \%$ was slightly higher in the ACS group, although this difference was not significant $(p=0.34)$. There was also no difference in MLA between the groups in our patient cohort ( $6.2 \pm 2.1$ vs. $5.6 \pm 1.5 ; p=0.21)$. Although IVUS criteria may not automatically be applied to BVS in every case, we believe that the parameters that are generally accepted for the assessment of adequate deployment point towards a good acute result as they are above the published reference values in both groups, especially if it is taken into account that OCT has been shown to measure lower absolute areas than IVUS [14].

OCT analysis did not show a significantly different post-procedural scaffold geometry. Assessment of the acute geometric parameters depicted a mean eccentricity index of $0.78 \pm 0.13$ and $0.78 \pm 0.06$ for the non-ACS and ACS groups, respectively. The clinical impact of asymmetric expansion has been evaluated in the MUSIC trial [15]. Here, first-generation DES with an eccentricity value of 0.7 were associated with favourable angiographic results at the 6-month follow-up. Furthermore, the impact of post-procedural eccentricity of Absorb BVS has been studied as part of the ABSORB II trial [16]. Suwannasom et al. found that BVS in general are more frequently associated with asymmetric and eccentric morphology than DES. This finding was independently associated with higher event rates. The symmetry index provides additional insight into the shape of the BVS; if the index is near zero, the BVS is symmetric throughout the entire length [17]. Both the eccentricity and symmetry index in our cohort demonstrated a good geometric shape, and the results are consistent with those reported in the literature $[17,18]$.

Tissue may prolapse through the scaffold struts after the implantation of BVS. Hypothetically, and according to the nature of thrombotic occlusion of a vessel (at least in patients with STEMI), one would expect more thrombotic tissue to prolapse in patients with ACS. However, we did not observe a larger prolapse area in our group of ACS patients. It has to be noted that post-dilatation balloons and pressure inflation did not differ significantly between the groups. Nevertheless, there was a slight tendency for more tissue in the ACS group that might not have reached significance due to the proportion of STEMI patients enrolled (35.3\%). Diletti et al. were able to show that BVS are able to entrap more thrombus due to their structure [19]. The clinical relevance of prolapse area in the long run is unclear, but in the short term this is of importance as the magnitude of prolapse area is related to increased post-procedural myocardial injury and elevation of CK-MB (creatine kinase) [20].

It was previously assumed that malapposition can lead to stent-related effects and increase adverse event rates [21], but only recently was malapposition identified as a predictor of late and very late ScT in patients treated with BVS [22]. This was irrespective of the timing of the finding during follow-up. Malapposed struts may disrupt laminar flow and activate platelets due to high shear stress [23], thereby contributing to the multifactorial aetiology of ScT. Accordingly, previous studies were able to show that usage of IVUS and OCT to guide the implantation process of DES can reduce event rates [24, 25 ]. ISA rates of $2 \%$ have been reported in the literature [18]. However, in the prospective, multi-centre PRAGUE 19 study, OCT analysis revealed only $1.1 \%$ malapposed struts, a much lower proportion than observed in the Absorb Cohort B study $(3.5 \%)[26,27]$. The rate of ISA in our cohort was $2.7 \%$ in patients who were treated with chronic coronary syndrome and $1.9 \%$ in those treated due to ACS. Although these values are not statistically different, the numerically slightly higher frequency in the non-ACS group is consistent with the finding of more fractures in the non-ACS group because it has to be assumed that the underlying plaque morphology and especially composition may have a negative influence on the expansion and apposition capabilities and may also favour strut discontinuity of BVS. This is supported by Shaw et al., who were able to demonstrate that expansion and eccentricity of Absorb BVS are significantly impacted by coronary artery plaque composition, morphology, and burden [28]. On the other hand, our group was able to show that this dependence is not always applicable to DESolve BVS [29]. The reason for this discrepancy may be a rigorous lesion preparation but may also be the ability of the DESolve BVS to self-correct for minor malappo- 
sition. Furthermore, Ormiston et al. performed a bench test that showed the safe threshold for post-dilatation with an Absorb BVS 3.0 to be $3.8 \mathrm{~mm}$ at $20 \mathrm{~atm}$, whereas it was $5.0 \mathrm{~mm}$ with $20 \mathrm{~atm}$ for the DESolve $3.0 \mathrm{~mm}$ [7]. Overall, this might have contributed to the lack of significance with respect to the observation of fractures and malapposed struts between the two groups in the present cohort and may suggest an inferior mechanical performance of BVS in stable lesions despite similar indices. This observation is consistent with the findings of Baquet et al., who analysed 26 consecutive patients with OCT 6-8 weeks after BVS implantation (46\% ACS) [30]. They reported a numerically higher proportion of protruding and malapposed struts in patients with chronic coronary syndrome than in patients with ACS: protruding $1.26 \%$ vs. $4.3 \%$; malapposed $0.39 \%$ vs. $2.48 \%$, respectively. On the other hand, they did not observe any difference in the amount of strut coverage or in the proportion of uncovered scaffolds between ACS and chronic coronary syndrome patients.

There are single- and multi-centre observational registries and also randomized trials that assessed BVS performance in ACS patients. The Mainz ACS registry compared 150 patients treated with BVS vs. a control group that was treated with DES during the same period and reported similarly favourable outcomes [31]. In the first propensity matching comparison of BVS in STEMI patients, the major adverse cardiovascular event (MACE) rate in the BVS group was higher than in the DES group [32]. However, the use of an early implantation technique contributed substantially to the event rates, as they were reduced with increasing experience: ScT occurred primarily in the acute phase and in patients without post-dilatation. Imori et al. confirmed the relevance of post-dilatation in ACS settings [33]. The first randomized trial including ACS patients was the EVERBIO II trial [34]. Patients (39\% presenting with ACS) were randomly assigned to a BVS or an everolimus-eluting stent (Promus Element; Boston Scientific, Marlborough, Massachusetts, USA) or a biolimus-eluting stent (Biomatrix Flex, Biosensors Europe SA, Morges, Switzerland). The patient- and device-oriented endpoints did not differ after 9 months. No definite stent thrombosis was reported. In addition, the TROFI II trial focused solely on STEMI patients [35]. One case of definite sub-acute ScT occurred in the BVS group ( $1.1 \%$ vs. $0 \% ; p=n s)$. These data are consistent with our findings, as none of the patients in our cohort showed an adverse event within the ensuing post-procedural period. In particular, no ScT was documented.

While initial studies showed comparable outcomes for BVS and DES, meta-analyses raised concerns regarding adverse events due to higher early and late thrombogenicity of BVS. Ultimately, the Absorb BVS was withdrawn from the market. Elixir Medical Corporation adapted and followed this decision with the PLLA-based DESolve BVS. However, a multicentre post-market analy- sis showed reasonable follow-up data with an ScT rate of $1 \%$ [36]. This may be explained by a favourable acute mechanical result that seems to be less dependent on the underlying lesion morphology, as shown previously by our study group [29]. This is the first study showing a feasible acute mechanical performance of DESolve BVS with OCT irrespective of the initial clinical presentation. New BVS platforms are being developed due to the lingering desire for BVS with transient vessel support. They are currently under clinical investigation [37, 38]. The present study supports this development by contributing reasonable mechanical outcome data for patients presenting with acute and chronic coronary syndrome. Especially patients presenting with ACS represent an important cohort and should not be excluded from large, randomized trials in the future.

There are several limitations associated with this study. Its registry nature with retrospective collection of the patients' data has inherent limitations and the evidence provided should be seen as hypothesis generating. In addition, the DESolve BVS is no longer available as Elixir Medical Corporation followed the decision of Abbott Vascular, which took the Absorb BVS off the market due to the reported adverse clinical events. Furthermore, although the protocols used for lesion preparation, scaffold deployment, and post-dilatation were the same for all operators contributing to this study, their adaptation due to the operator's decision may have affected the final acute mechanical result and cannot be excluded. Furthermore, the sample size of the study was small, and we did not perform an analysis of the plaque morphology, which might have helped to understand the increasing mechanical discontinuities seen in the non-ACS group. Further randomized clinical trials are required to investigate the use of BVS in the setting of acute and chronic coronary syndrome, ideally with broad application of intravascular imaging.

\section{Conclusions}

In randomized trials mid- and long-term outcomes of $\mathrm{PCl}$ procedures with BVS have been assessed predominantly in patients with stable coronary disease, and relatively simple lesions and patients presenting with ACS were excluded, although these patients in particular might benefit from the BVS platform. We were able to show that the clinical presentation does not have an effect on acute mechanical performance of a Novolimus-eluting BVS as assessed by OCT.

\section{Acknowledgments}

We are grateful to Elizabeth Martinson, Ph.D., of the KHFI Editorial Office, for providing editorial assistance.

\section{Conflict of interest}

The authors declare no conflict of interest. 


\section{References}

1. Serruys PW, Garcia-Garcia HM, Onuma Y. From metallic cages to transient bioresorbable scaffolds: change in paradigm of coronary revascularization in the upcoming decade? Eur Heart $J$ 2012; 33: 16-25.

2. Serruys PW, Chevalier B, Sotomi Y, et al. Comparison of an everolimus-eluting bioresorbable scaffold with an everolimus-eluting metallic stent for the treatment of coronary artery stenosis (ABSORB II): a 3 year, randomised, controlled, single-blind, multicentre clinical trial. Lancet 2016; 388: 2479-91.

3. Wykrzykowska J, Kraak RP, Hofma SH, et al. Bioresorbable scaffolds versus metallic stents in routine PCI. N Engl J Med 2017; 376: 2319-28.

4. Capodanno D, Gori T, Nef H, et al. Percutaneous coronary intervention with everolimus-eluting bioresorbable vascular scaffolds in routine clinical practice: early and midterm outcomes from the European multicentre GHOST-EU registry. Eurolntervention 2015; 10: 1144-53.

5. Nef HM, Wiebe J, Kastner J, et al. Everolimus-eluting bioresorbable scaffolds in patients with coronary artery disease: results from the German-Austrian ABSORB ReglstRy (GABI-R). Eurolntervention 2017; 13: 1311-8

6. Kawamoto H, Ruparelia N, Tanaka A, et al. Bioresorbable scaffolds for the management of coronary bifurcation lesions. JACC Cardiovasc Interv 2016; 9: 989-1000.

7. Ormiston JA, Webber B, Ubod B, et al. An independent bench comparison of two bioresorbable drug-eluting coronary scaffolds (Absorb and DESolve) with a durable metallic drug-eluting stent (ML8/Xpedition). Eurolntervention 2015; 11: 60-7.

8. Wiebe J, Bauer T, Dörr O, et al. Implantation of a novolimus-eluting bioresorbable scaffold with a strut thickness of $100 \mu \mathrm{m}$ showing evidence of self-correction. Eurointervention 2015; 11: 204.

9. Ryan TJ, Faxon DP, Gunnar RM, et al. Guidelines for percutaneous transluminal coronary angioplasty. A report of the American College of Cardiology/American Heart Association Task Force on Assessment of Diagnostic and Therapeutic Cardiovascular Procedures (Subcommittee on Percutaneous Transluminal Coronary Angioplasty). Circulation 1988; 78: 486-502.

10. Tamburino C, Latib A, van Geuns RJ, et al. Contemporary practice and technical aspects in coronary intervention with bioresorb able scaffolds: a European perspective. Eurolntervention 2015; 11: 45-52.

11. Uren NG, Schwarzacher SP, Metz JA, et al. Predictors and outcomes of stent thrombosis: an intravascular ultrasound registry. Eur Heart J 2002; 23: 124-32.

12. Sonoda S, Morino Y, Ako J, et al. Impact of final stent dimensions on long-term results following sirolimus-eluting stent implantation: serial intravascular ultrasound analysis from the sirius trial. J Am Coll Cardiol 2004; 43: 1959-63.

13. Fujii K, Carlier SG, Mintz GS, et al. Stent underexpansion and residual reference segment stenosis are related to stent thrombosis after sirolimus-eluting stent implantation: an intravascular ultrasound study. J Am Coll Cardiol 2005; 45: 995-8.

14. Gonzalo N, Serruys PW, García-García HM, et al. Quantitative ex vivo and in vivo comparison of lumen dimensions measured by optical coherence tomography and intravascular ultrasound in human coronary arteries. Rev Esp Cardiol 2009; 62; 615-24.

15. de Jaegere P, Mudra H, Figulla $\mathrm{H}$, et al. Intravascular ultrasound-guided optimized stent deployment. Immediate and 6 months clinical and angiographic results from the Multicenter
Ultrasound Stenting in Coronaries Study (MUSIC Study). Eur Heart J 1998; 19: 1214-23.

16. Suwannasom P, Sotomi $Y$, Ishibashi $Y$, et al. The impact of post-procedural asymmetry, expansion, and eccentricity of bioresorbable everolimus-eluting scaffold and metallic everolimus-eluting stent on clinical outcomes in the ABSORB II trial. JACC Cardiovasc Interv 2016; 9: 1231-42.

17. Brugaletta S, Gomez-Lara J, Diletti R, et al. Comparison of in vivo eccentricity and symmetry indices between metallic stents and bioresorbable vascular scaffolds: insights from the ABSORB and SPIRIT trials. Catheter Cardiovasc Interv 2012; 79: 219-28.

18. Mattesini A, Secco GG, Dall'Ara G, et al. ABSORB biodegradable stents versus second-generation metal stents: a comparison study of 100 complex lesions treated under OCT guidance. JACC Cardiovasc Interv 2014; 7: 741-50.

19. Diletti R, van der Sijde J, Karanasos A, et al. Differential thrombotic prolapse burden in either bioresorbable vascular scaffolds or metallic stents implanted during acute myocardial infarction: the snowshoe effect: insights from the maximal footprint analysis. Int J Cardiol 2016; 220: 802-8.

20. Sugiyama T, Kimura S, Akiyama D, et al. Quantitative assessment of tissue prolapse on optical coherence tomography and its relation to underlying plaque morphologies and clinical outcome in patients with elective stent implantation. Int J Cardiol 2014; 176: 182-90.

21. Ahn JM, Kang SJ, Yoon SH, et al. Meta-analysis of outcomes after intravascular ultrasound-guided versus angiography-guided drug-eluting stent implantation in 26,503 patients enrolled in three randomized trials and 14 observational studies. Am J Cardiol 2014; 113: 1338-47.

22. Boeder NF, Weissner M, Blachutzik F, et al. Incidental finding of strut malapposition is a predictor of late and very late thrombosis in coronary bioresorbable scaffolds. J Clin Med 2019; 8: 580.

23. Gomez-Lara J, Radu M, Brugaletta $S$, et al. Serial analysis of the malapposed and uncovered struts of the new generation of everolimus-eluting bioresorbable scaffold with optical coherence tomography. JACC Cardiovasc Interv 2011; 4: 992-1001.

24. Witzenbichler B, Maehara A, Weisz G, et al. Relationship between intravascular ultrasound guidance and clinical outcomes after drug-eluting stents: the assessment of dual antiplatelet therapy with drug-eluting stents (ADAPT-DES) study. Circulation 2014; 129: 463-70.

25. Prati F, Romagnoli E, Burzotta F, et al. Clinical impact of OCT findings during PCl: the CLI-OPCI II study. JACC Cardiovasc Imaging 2015; 8: 1297-305.

26. Kočka V, Malý M, Toušek P, et al. Bioresorbable vascular scaffolds in acute ST-segment elevation myocardial infarction: a prospective multicentre study 'Prague 19'. Eur Heart J 2014; 35: 787-94.

27. Serruys PW, Ormiston JA, Onuma Y, et al. A bioabsorbable everolimus-eluting coronary stent system (ABSORB): 2-year outcomes and results from multiple imaging methods. Lancet 2009; 373: 897-910.

28. Shaw E, Allahwala UK, Cockburn JA, et al. The effect of coronary artery plaque composition, morphology and burden on Absorb bioresorbable vascular scaffold expansion and eccentricity a detailed analysis with optical coherence tomography. Int J Cardiol 2015; 184: 230-6.

29. Boeder NF, Dörr O, Bauer T, et al. Effect of plaque composition, morphology, and burden on DESolve novolimus-eluting bioresorbable vascular scaffold expansion and eccentricity - an op- 
tical coherence tomography analysis. Cardiovasc Revasc Med 2019; 20; 480-4.

30. Baquet M, Brenner C, Wenzler M, et al. Impact of clinical presentation on early vascular healing after bioresorbable vascular scaffold implantation. J Interv Cardiol 2017; 30: 16-23.

31. Gori T, Schulz E, Hink U, et al. Early outcome after implantation of Absorb bioresorbable drug-eluting scaffolds in patients with acute coronary syndromes. Eurointervention 2014; 9: 1036-41.

32. Fam JM, Felix C, van Geuns RJ, et al. Initial experience with everolimus-eluting bioresorbable vascular scaffolds for treatment of patients presenting with acute myocardial infarction: a propensity-matched comparison to metallic drug eluting stents 18-month follow-up of the BVS STEMI first study. Eurointervention 2016; 12: 30-7.

33. Imori Y, D'Ascenzo F, Gori T, et al. Impact of postdilatation on performance of bioresorbable vascular scaffolds in patients with acute coronary syndrome compared with everolimus-eluting stents: a propensity score-matched analysis from a multicenter "real-world" registry. Cardiol J 2016; 23: 374-83.

34. Puricel S, Arroyo D, Corpataux N, et al. Comparison of everolimus- and biolimus-eluting coronary stents with everolimus-eluting bioresorbable vascular scaffolds. J Am Coll Cardiol 2015; 65: 791-801.

35. Sabaté $M$, Windecker $S$, Iñiguez $A$, et al. Everolimus-eluting bioresorbable stent vs. durable polymer everolimus-eluting metallic stent in patients with ST-segment elevation myocardial infarction: results of the randomized ABSORB ST-segment elevation myocardial infarction-TROFI II trial. Eur Heart J 2016; 37: 229-40.

36. Nef $\mathrm{H}$, Wiebe J, Boeder N, et al. A multicenter post-marketing evaluation of the Elixir DESolve $\left({ }^{\circledR}\right)$ Novolimus-eluting bioresorbable coronary scaffold system: first results from the DESolve PMCF study. Catheter Cardiovasc Interv 2018; 92: 1021-7.

37. Arroyo D, Gendre G, Schukraft S, et al. Comparison of everolimus- and biolimus-eluting coronary stents with everolimus-eluting bioresorbable vascular scaffolds: two-year clinical outcomes of the EVERBIO II trial. Int J Cardiol 2017; 243: 121-5.

38. Chevalier B, Abizaid A, Carrié D, et al. Clinical and angiographic outcomes with a novel radiopaque sirolimus-eluting bioresorbable vascular scaffold. Circ Cardiovasc Interv 2019; 12: e007283. 